\title{
Comparative genome analysis of pathogenic and non-pathogenic Clavibacter strains reveals adaptations to their lifestyle
}

Joanna Załuga ${ }^{*}$, Pieter Stragier ${ }^{1}$, Steve Baeyen², Annelies Haegeman², Johan Van Vaerenbergh², Martine Maes ${ }^{2}$ and Paul De Vos ${ }^{1,3}$

\begin{abstract}
Background: The genus Clavibacter harbors economically important plant pathogens infecting agricultural crops such as potato and tomato. Although the vast majority of Clavibacter strains are pathogenic, there is an increasing number of non-pathogenic isolates reported. Non-pathogenic Clavibacter strains isolated from tomato seeds are particularly problematic because they affect the current detection and identification tests for Clavibacter michiganensis subsp. michiganensis $(\mathrm{Cmm})$, which is regulated with a zero tolerance in tomato seed. Their misidentification as pathogenic $\mathrm{Cmm}$ hampers a clear judgment on the seed quality and health.
\end{abstract}

Results: To get more insight in the genetic features linked to the lifestyle of these bacteria, a whole-genome sequence of the tomato seed-borne non-pathogenic Clavibacter LMG 26808 was determined. To gain a better understanding of the molecular determinants of pathogenicity, the genome sequence of LMG 26808 was compared with that of the pathogenic Cmm strain (NCPPB 382). The comparative analysis revealed that LMG 26808 does not contain plasmids pCM1 and pCM2 and also lacks the majority of important virulence factors described so far for pathogenic $\mathrm{Cmm}$. This explains its apparent non-pathogenic nature in tomato plants. Moreover, the genome analysis of LMG 26808 detected sequences from a plasmid originating from a member of Enterobacteriaceae/Klebsiella relative. Genes received that way and coding for antibiotic resistance may provide a competitive advantage for survival of LMG 26808 in its ecological niche. Genetically, LMG 26808 was the most similar to the pathogenic Cmm NCPPB 382 but contained more mobile genetic elements. The genome of this non-pathogenic Clavibacter strain contained also a high number of transporters and regulatory genes.

Conclusions: The genome sequence of the non-pathogenic Clavibacter strain LMG 26808 and the comparative analyses with other pathogenic Clavibacter strains provided a better understanding of the genetic bases of virulence and adaptation mechanisms present in the genus Clavibacter.

Keywords: Non-pathogenic Clavibacter, Bacterial wilt and canker, Tomato seeds, Genome sequencing, Quarantine, Plant pathogen

\section{Background}

Clavibacter is generally considered a genus of plant pathogens, but ecological surveys suggest that environmental, non-pathogenic Clavibacter occur more commonly than previously thought $[1,2]$. Generally these non-pathogenic isolates are overlooked since diagnostic procedures focus on pathogenic strains. Just recently, studies were undertaken to

\footnotetext{
*Correspondence: Joanna.Zaluga@ugent.be

'Laboratory of Microbiology, Department of Biochemistry and Microbiology, Ghent University, K.L. Ledeganckstraat 35, Gent B-9000, Belgium

Full list of author information is available at the end of the article
}

initiate the characterization of these non-pathogenic isolates $[3,4]$. The main objective of this study was to investigate key genomic features of non-pathogenic Clavibacter isolated from tomato seeds. These strains tend to be misidentified as $\mathrm{Cmm}$ in serological and molecular tests used in seed assays. Their high genetic and phenotypic similarity to pathogenic $\mathrm{Cmm}$ strains hampers a clear judgment on seed health.

The majority of non-pathogenic Clavibacter strains isolated from tomato seeds exhibit similar cell and colony morphology to the genuine $\mathrm{Cmm}$ [5]. Because of

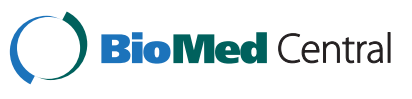


the common biological origin (tomato seed), high sequence similarities and similar physiological characteristics, the non-pathogenic Clavibacter strains are suggested to be the most related to $\mathrm{Cmm}$. Initial in planta experiments demonstrated that this group of isolates is not pathogenic to the tomato plant and they do not colonize the vascular tissues of tomato [4]. Non-pathogenic clavibacters neither induce a hypersensitive reaction (HR) after infiltration of Nicotiana tabacum and Nicotiana benthamiana leaves [3], nor when inoculated to Mirabilis jalapa (J. Van Vaerenbergh, personal communication). Furthermore, a majority of these strains is lacking one or both $\mathrm{Cmm}$ plasmids carrying important virulence factors.

So far there is very little information available on nonpathogenic Clavibacter strains isolated from tomato seeds. Reports concerning the ecological niche, survival abilities or nutritional requirements are lacking. Knowledge about the biology of these strains is limited, not only because they were only recently identified as constituting a separate Clavibacter group but also because their significance in the Cmm identification procedure has not been evaluated previously. Their ecological niches remain unknown; routes of transmission and possible sources of these strains have not yet been recognized.

High genetic and phenotypic similarities of nonpathogenic Clavibacter and pathogenic $\mathrm{Cmm}$ strains are the main reasons for their misidentifications as $\mathrm{Cmm}$ in the currently recommended detection/identification tests for $\mathrm{Cmm}$ in tomato seeds [6]. Cross-reactions with antisera specific for $\mathrm{Cmm}$ and/or positive PCR reactions with primers used for identification of $\mathrm{Cmm}$ illustrate the proximity of surface antigens and genomic sequences of non-pathogenic seed-borne Clavibacter to the pathogenic $\mathrm{Cmm}$ [3]. Recent studies demonstrated that neither PCR assays based on commonly used $16 \mathrm{~S}$ rRNA genes or ITS region, nor those designed for the detection of known virulence factors are specific to only pathogenic $\mathrm{Cmm}$ [3,7]. Furthermore, some non-pathogenic Clavibacter strains showed fainter PCR amplicons on the gel impeding the correct interpretation of the results [4]. Taxonomically, these non-pathogenic clavibacters from tomato seeds are distinct from all Clavibacter subspecies (based on the analysis of housekeeping genes gyrB and $\operatorname{dnaA}$ ) [4].

Recent developments in the field of molecular biology and sequencing allowed generating complete genome sequences and subsequently determining metabolic traits for many organisms. Complete genome sequences of $\mathrm{Cmm}$ NCPPB 382 [8], Cms ATCC 33113 [9] and Cmn NCPPB 2581 (released without publication) provide genetic information that allows for comparative studies and helps to better understand their pathogenicity characteristics and host adaptation. However, no information is available about the genome content of non-pathogenic Clavibacter strains, which could deliver some informative insights into the differences in virulence determinants, genetic content and adaptation to a lifestyle in their natural ecological niche(s). Genome comparison between pathogenic and non-pathogenic strains belonging to the same species is an important and valuable approach to identify genes that may contribute to virulence and general fitness of the organism.

In this report we present the genome analysis of nonpathogenic Clavibacter LMG 26808 isolated from tomato seed. The specific purposes of this study were a) to generate a draft genome sequence of this strain, b) to analyze it for virulence-related gene content by comparing it to the available genome of the pathogenic Clavibacter michiganensis subsp. michiganensis (Cmm) NCPPB 382 , c) to perform a comparative analysis with the genomes of Cmm (NCPPB 382) [8], Clavibacter michiganensis subsp. nebraskensis (Cmn) (NCPPB 2581, released without publication) and Clavibacter michiganensis subsp. sepedonicus (Cms) (ATCC 33113) [9], pathogenic to tomato, maize and potato, respectively, d) to search for adaptations to a non-pathogenic lifestyle.

\section{Methods}

\section{Strains and DNA extraction}

Non-pathogenic Clavibacter sp. LMG 26808 was received as isolate PD 5684 from Naktuinbouw, The Netherlands. It was obtained in dilution plating on semi-selective media according to the current method for detection of $\mathrm{Cmm}$ in tomato seeds recommended by the International Seed Federation (ISF) [6]. LMG 26808 is phenotypically similar to $\mathrm{Cmm}$ on SCMF and CMM1T and was identified as $\mathrm{Cmm}$ in commonly practiced PCR tests but showed no pathogenicity in tomato plants [3,4]. LMG 26808 was aerobically grown on MTNA (mannitol, trimethoprim, nalidixic acid, amphotericin) medium without antibiotics at $25^{\circ} \mathrm{C}$ for $24 \mathrm{~h}-48 \mathrm{~h}$ [10]. Stock cultures were stored at $-80^{\circ} \mathrm{C}$ in Microbank ${ }^{\mathrm{TM}}$ beads (Pro-Lab Diagnostics, Canada). Total genomic DNA was extracted according to the guanidium-thiocyanate-EDTA-sarkosyl method described by Pitcher [11], which was adapted for Grampositive bacteria by a pre-treatment with lysozyme ( $5 \mathrm{mg} / \mu \mathrm{l}$ lysozyme in TE buffer) and incubation for 40 minutes at $37^{\circ} \mathrm{C}$.

\section{Plasmid extraction}

Isolation of plasmid DNA was based on the alkaline method of Anderson and McKay [12]. Agarose gel electrophoresis was performed in a Tris acetate buffer containing $40 \mathrm{mM}$ Tris, $20 \mathrm{mM}$ acetic acid, and $2 \mathrm{mM}$ $\mathrm{Na}_{2}$ EDTA ( $\mathrm{pH}$ 8.1). Gels contained $0.8 \%$ agarose and electrophoresis was performed at $55 \mathrm{~V}$ for $16 \mathrm{hrs}$ at $4^{\circ} \mathrm{C}$. Gels were stained with ethidium bromide $0.5 \mu \mathrm{g} / \mathrm{ml}$. 


\section{Genome sequencing}

Library preparation and genome sequencing was performed by BaseClear (Leiden, The Netherlands). Highmolecular weight genomic DNA was used as input for library preparation using the Illumina TruSeq DNA library preparation kit (Illumina). Briefly, the gDNA was fragmented and subjected to end-repair, A-tailing, ligation of adaptors including sample-specific barcodes and sizeselection to obtain a library with median insert-size around $300 \mathrm{bp}$. After PCR enrichment, the resultant library was checked on a Bioanalyzer (Agilent) and quantified. The libraries were multiplexed, clustered, and sequenced on an Illumina HiSeq 2000 with pairedend 50 cycles protocol. The sequencing run was analyzed with the Illumina CASAVA pipeline (v1.8.2). The raw sequencing data produced was processed removing the sequence reads which were of too low quality (only "passing filter" reads were selected) and discarding reads containing adaptor sequences or PhiX control with an inhouse filtering protocol.

A paired-end (PE) DNA library with a mean insert size of $300 \mathrm{bp}$ was sequenced with average reads of $101 \mathrm{bp}$ on an Illumina Genome HiSeq2000 (Illumina Inc.). Next, a mate-paired (MP) DNA library with a mean insert size of $3800 \mathrm{bp}$ was sequenced with average reads of $51 \mathrm{bp}$ on an Illumina Hiseq2000 (Illumina Inc.). Automatic trimming (based on a threshold of $Q=20$ ) and assembly was performed using CLC Genomics Workbench v5.0. An initial de novo assembly was performed in CLC Genomics Workbench v5.0 using the quality trimmed and paired reads from the PE and MP reads. All contigs shorter than 200 bp were discarded. Remaining $\mathrm{N}$-nucleotides in the scaffolds, introduced during scaffolding, were removed from the final sequence by breaking up the scaffolds back into contigs where they were encountered. The quality of the final draft genome sequence was compared to the initial PE-based de novo assembly through comparative read-mapping in CLC Genomics Workbench v5.0 using the trimmed read sets. Contigs were ordered automatically with MAUVE [13] and manually with Artemis [14] by comparing with Cmm NCPPB 382.

\section{Genome annotation}

Functional annotation and metabolic reconstruction were performed with (1) the Rapid Annotation Subsystem Technology (RAST) server [15], using Glimmer [16] for gene calling and allowing frameshift correction, backfilling of gaps and automatic fixing of errors, with (2) the Integrated Microbial Genomes Expert Review (IMG-ER) annotation pipeline (https://img.jgi.doe.gov/cgi-bin/er/main. cgi) [17]. Assigned functions were checked with BLAST [18]. Alignment and phylogenetic analysis were performed with MEGA 5.0 [19].

\section{Comparative genomic analysis}

Artemis software was used for data management and DNAPlotter was used for genome visualization [20]. The MAUVE alignment tool was used for multiple genomes sequence alignment and visualization. IslandViewer was used to analyze possible genomic islands (GI) on the draft genome [21]. IslandViewer integrates two sequence composition GIs prediction methods, namely IslandPathDIMOB [22] and SIGI-HMM [23] and one single comparative GI prediction method, namely IslandPick [21] for genomic island prediction.

ISsaga application from ISfinder server [24] was used to identify insertion sequences (IS) and transposons in the draft genome of LMG 26808. Sequences exhibiting homology to IS and transposon sequences were verified with the Mobilomics software [25]. The core genome was estimated using the Phylogenetic profiler tool that is part of the IMG system (https://img.jgi.doe.gov/cgi-bin/ er/main.cgi) at a similarity cutoff of $50 \%$.

The presence of possible virulence-related genes and genes expressed during tomato infection in the draft genome of the non-pathogenic Clavibacter was analyzed by comparing it with tomato pathogen $\mathrm{Cmm} \mathrm{NCPPB}$ 382. The comparative screening of the gene content was performed in RAST, IMG-ER and EDGAR [26]. Absence or presence of coding sequences in each genome, as reported by RAST, IMG-ER and EDGAR were independently confirmed by performing protein and nucleotide BLAST queries in the target genomes. Proteins with amino acid sequence similarities higher than $50 \%$ and with a coverage higher than $70 \%$ were considered homologs. Based on the RAST, IMG-ER and EDGAR annotation results, the presence of known and putative virulence factors, pathogenicity related genes and genes uniquely present in the non-pathogenic Clavibacter LMG 26808 were investigated. Identification of orthologous groups between four available Clavibacter genomes was achieved by OrthoMCL analyses [27]. OrthoMCL clustering analyses were performed using default parameters with the P-value Cut-off $=1 \times 10^{-5}$.

\section{Deposition}

The current draft genome sequence was deposited at Genbank under accession number AZQZ00000000 after automatic annotation by the PGAAP online annotation pipeline.

\section{Results and discussion}

\section{General features of non-pathogenic Clavibacter LMG 26808}

Genome assembly using paired-end and mate-paired reads resulted in a $3.47 \mathrm{Mb}$ sequence represented in 70 contigs from which the longest covered more than one million bp (Table 1). The initial PE de novo assembly 
Table 1 Genome characteristics of the non-pathogenic Clavibacter LMG 26808

\begin{tabular}{lc}
\hline Genome characteristics & $\begin{array}{c}\text { Non-pathogenic } \\
\text { Clavibacter LMG 26808 }\end{array}$ \\
\hline No. contigs (>200 bp) & 70 \\
Total contig size (bp) & $3,476,455$ \\
N50 (bp) after scaffolding & 383,456 \\
Largest contig size (bp) & $1,028,177$ \\
GC content (\%) & 72.01 \\
No. RNA calls & 7 rRNA \\
& 46 tRNA \\
No. CDS calls & 3218 \\
NCBI accession no. & AZQZ00000000 \\
Number of insertion elements & 10 \\
\hline
\end{tabular}

was used for scaffolding with the MP dataset. In the final consensus sequence each base matched at least Phred quality score of 35 . LMG 26808 contains one chromosome and evidence of a presence of a plasmid that showed a high similarity to a Klebsiella pneumoniae Kp11978 plasmid pOXA-48 (JN626286.1). The genes of Kp11978 were found on 15 contigs in a draft genome of LMG 26808 (estimated size of these contigs $\sim 48 \mathrm{kbp}, \% \mathrm{GC} \sim 50 \%$ ) (Additional file 1: Table S1). No sequences of known Clavibacter plasmids could be detected. The GC content of the draft genome averages $72 \%$. There are 46 tRNA genes and two complete rRNA operons. A total of 3218 protein-coding genes are predicted in non-pathogenic Clavibacter strain (in IMGER), which is similar to the $\mathrm{Cmm}$ genome NCPPB 382 that contains 3107 protein-coding genes. The genome of the non-pathogenic Clavibacter strain contains 685 (21.3\%) proteins without predicted function being either annotated as conserved hypothetical proteins or proteins with unknown function.

The number of genes detected in the draft genome of LMG 26808 was higher than in the other three complete Clavibacter genomes (Table 2). The average nucleotide identity (ANI) between the draft genome of the nonpathogenic Clavibacter and the three published Clavibacter genomes Cmm NCPPB 382 (NC_009480.1), Cms ATCC 33113 (NC_010407.1) and Cmn NCPPB 2581 (NC_020891.1) was determined using the in silico DNADNA hybridization (DDH) method included in the software JSpecies [28]. The results indicated that LMG 26808 is genetically most related to Cmm NCPPB 382 (94.96\% ANI), followed by Cmn NCPPB 2581 (92.75\% ANI) and Cms ATCC 33113 (92.48\% ANI). Although based on the ANI values the LMG 26808 genome is the most similar to that of pathogenic Cmm NCPPB 382, the synteny plots of LMG 26808 and Cmn NCPPB 2581 and

Table 2 Comparison of genome characteristics (based on IMG-ER server)

\begin{tabular}{|c|c|c|c|c|}
\hline Genome Name & $\begin{array}{l}\text { Clavibacter michiganensis } \\
\text { subps. michiganensis } \\
\text { NCPPB } 382\end{array}$ & $\begin{array}{c}\text { Clavibacter michiganensis } \\
\text { subsp. sepedonicus } \\
\text { ATCC } 33113\end{array}$ & $\begin{array}{l}\text { Clavibacter michiganensis } \\
\text { subsp. nebraskensis } \\
\text { NCPPB } 2581\end{array}$ & $\begin{array}{c}\text { Non-pathogenic } \\
\text { ClavibacterLMG } 26808\end{array}$ \\
\hline Accession number $^{a}$ & NC_009480.1 & NC_010407.1 & NC_020891.1 & AZQZ000000000 \\
\hline Host & tomato & potato & maize & * \\
\hline Disease & bacterial wilt and canker & potato ring rot & wilt and blight & non-pathogenic \\
\hline Size & 3395237 & 3403786 & 3063596 & 3476455 \\
\hline Genes & 3169 & 3168 & 2936 & 3282 \\
\hline CDS & 3107 & 3117 & 2890 & 3218 \\
\hline CDS (\%) & 98.04 & 98.39 & 98.43 & 98.05 \\
\hline RNA & 62 & 51 & 46 & 64 \\
\hline rRNA & 6 & 6 & 6 & 7 \\
\hline tRNA & 45 & 45 & 30 & 46 \\
\hline Enzymes & 759 & 712 & 740 & 750 \\
\hline CRISPR & 1 & 0 & 0 & 1 \\
\hline GC\% & 72 & 72 & 73 & 72 \\
\hline Coding bases & 3041059 & 2955244 & 2823671 & 3074588 \\
\hline Signalp ${ }^{b}$ & 281 & 234 & 219 & 140 \\
\hline Signalp (\%) & 8.87 & 7.39 & 7.46 & 4.27 \\
\hline Homologs to LMG $26808(\%)^{c}$ & $2716(87.4)$ & $2457(78.8)$ & $2531(87.5)$ & - \\
\hline
\end{tabular}

${ }^{a}$ Only the Genbank records of the chromosomes are given.

${ }^{b}$ Number of genes coding signal peptides.

c Calculated using a Genome Gene Best Homologs tool included in IMG-ER server.

*Isolated from tomato seeds, host unknown. 
the percentage of homologous genes shared by LMG 26808 and Cmn NCPPB 2581 are also considerably high (Table 2, Figure 1). The genomes of LMG 26808, NCPPB 382 and NCPPB 2581 are collinear with less than 5 recombinational breakpoints.

Comparison on a genomic scale revealed a high conservation in the gene sequence among genomes of LMG 26808, NCPPB 382 and NCPPB 2581 (Figure 1). There are 299 genes $(\sim 10 \%)$ present in the LMG $26808 \mathrm{draft}$ genome that were not detected in the Cmm NCPPB 382 genome. Forty eight of them were detected in $\mathrm{Cmn}$ and/ or Cms genomes (Additional file 1: Table S2). 37 unique genes of LMG 26808 were associated with the plasmid and/or low GC regions. 214 of the unique genes were found in the core chromosome of LMG 26808 (Additional file 1: Table S2). Almost half of the genes specific for LMG 26808 belonged to hypothetical or unknown proteins (120). Remaining sequences were coding for additional $A B C$ transporters, antibiotic resistance genes, acetyltransferases and several enzymes that in majority could not be assigned to any KEGG (Kyoto Encyclopedia of Genes and Genomes) pathway (Additional file 1: Table S2). When compared to other Clavibacter genomes, LMG 26808 appeared to not have experienced gene losses and despite it is considered only a draft, the majority of important genes involved in basic metabolism and gene regulation could be detected. Comparative analysis (based on KEGG pathways) showed that LMG 26808 lacks sulfate and nitrate reduction pathways suggesting that its capability of survival in soli might be similar to this observed in Cmm NCPPB 382. The core genome consists of 2316 homologs found in all four Clavibacter genomes. LMG 26808 contains 12 genomic regions exhibiting a lower GC content (Additional file 1: Table S3). Several genes coding for proteins within these regions were found to contribute to the fitness of the bacterium (Cl_02679 coding for ABC-type $\mathrm{Fe}^{3+}$-siderophore transport system; Cl_03044 coding for permease component, chloramphenicol acetyltransferase (EC 2.3.1.28); Cl_03094 coding for multidrug-efflux transporter). The genome heterogeneity and genetic diversity among Clavibacter strains most likely contribute to the differences in the bacterial lifestyle. Phage-related recombinases (e.g. Cl_00892, Cl_03056), integrase/resolvase (e.g. Cl_02713) and other insertion elements (transposases, e.g. Cl_03190) associated with a phage were found in higher numbers in the genome of LMG 26808 than in the Cmm NCPPB 382 genome (Table 3). The genome of LMG 26808 contained sixteen genes coding for transposases and recombinases (Table 3). This number was much lower in comparison to more than 100 genes found in Cms (ATCC 33113) [9]. Detected IS belonged to IS3, IS4, IS5, IS6 and IS1380 families. Transposases were represented by $\operatorname{Tn} 3(20 \%)$. No pseudogenes among transposases and recombinases were detected suggesting that these elements may encode functional genes. None of the IS elements found in LMG 26808 has homologs in the other Clavibacter strains. The most common IS element in Cms ATCC 33113 is IS1121 [9]. Cmm NCPPB 382 contains only a few IS, which are most probably nonfunctional [8]. Cmn NCPPB 2581 contained only two types of IS, namely ILSre2 and ISNCY (predicted by ISsaga).

The comparison of functional categories as defined by COG (Clusters of Orthologous Groups) showed noticeable differences in the gene content in categories of 'carbohydrate transport and metabolism' and of 'translation, ribosomal structure and biogenesis'. All included Clavibacter strains contained a higher percentage of genes in these two categories than a free-living organism Escherichia coli $081 \mathrm{ED} 1 \mathrm{a}$ or a tomato pathogen as e.g. Pseudomonas syringae pv. tomato T1 (Additional file 1: Figure S1). These observations are supporting the hypothesis that compared Clavibacter strains can most probably utilize different sugars as an energy source and that they possess a wide range of transport systems that enable the efficient trafficking of the substrates and products. The presence of a high number of genes involved in translation, ribosomal structure and biogenesis implies that these bacteria respond more effectively and rapidly to nutritional resources, which can be an important advantage in a changing environment.
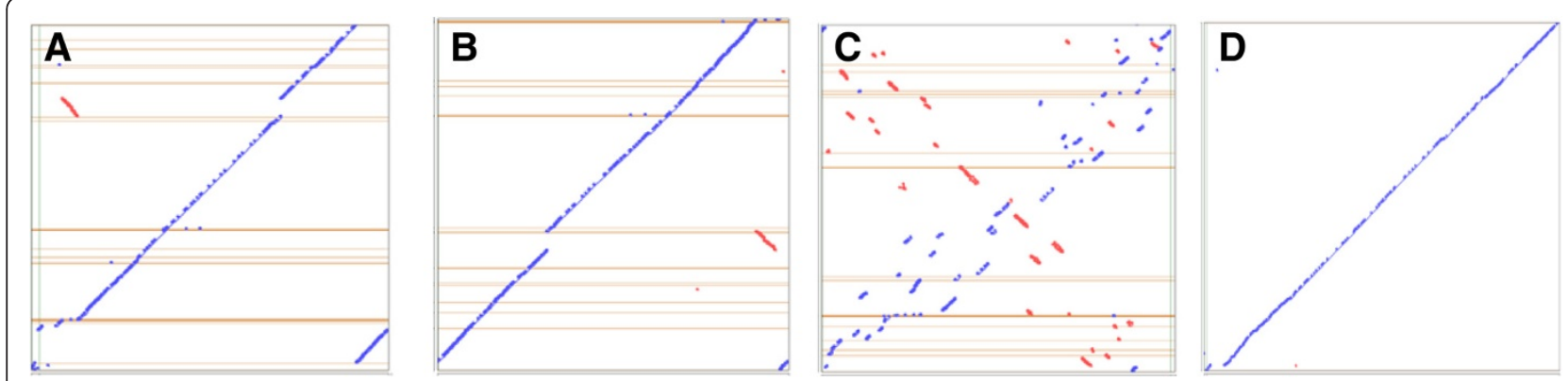

Figure 1 Syntenic dotplots showing the similarity of the genomes included in the analysis. A) non-pathogenic Clavibacter LMG 26808 ( $x$-axis) and Cmm NCPPB 382 (y-axis); B) non-pathogenic Clavibacter LMG 26808 (x-axis) and Cmn NCPPB 2581 (y-axis); C) non-pathogenic Clavibacter LMG 26808 (x-axis) and Cms ATCC 33113 (y-axis); D) Cmm NCPPB 382 (x-axis) and Cmn NCPPB 2581 (y-axis) (Diagrams generated in IMG-ER). 
Table 3 Mobile genetic elements found in the genome of LMG 26808 (Based on the annotation results from IMG-ER, RAST and EDGAR)

\begin{tabular}{|c|c|c|c|c|c|}
\hline CDS identifiers & COG & COG annotation & Pfam & Position & Length (bp) \\
\hline Cl_00892 & COG4974 & Site-specific recombinase XerD, phage_integrase & pfam00589 & Contig 3 (50199 to 51350) & 1152 \\
\hline Cl_00935 & COG4974 & Site-specific recombinase $X e r D$, phage_integrase & pfam00589 & Contig 5 (8554 to 9540) & 987 \\
\hline Cl_01562 & COG4974 & Site-specific recombinase XerD, phage_integrase & pfam00589 & Contig 5 (682570 to 683556) & 987 \\
\hline Cl_01811 & COG1842 & $\begin{array}{l}\text { Phage shock protein A (IM30), suppresses } \\
\text { sigma54-dependent transcription }\end{array}$ & pfam04012 & Contig 5 (938633 to 939376) & 744 \\
\hline Cl_01968 & COG3600 & Uncharacterized phage-associated protein & pfam13274 & contig 7 (7492 to 7956$)$ & 753 \\
\hline Cl_03043 & COG4679 & Phage-related protein & pfam05973 & contig 15 (974 to 1303) & 330 \\
\hline Cl_03056 & COG4974 & Site-specific recombinase $X e r D$, phage_integrase & pfam00589 & contig 15 (22760 to 23485) & 726 \\
\hline Cl_03252 & COG4974 & Site-specific recombinase $X e r D$, phage_integrase & pfam00589 & contig 46 (189 to 866$)$ & 678 \\
\hline Cl_02713 & COG2452 & Predicted site-specific integrase-resolvase & pfam12728 & contig 11 (350076 to 350522) & 447 \\
\hline Cl_03047 & COG2801 & Transposase and inactivated derivatives, Tnp1, IS3_IS150 & pfam01527 & contig 15 (9545 to 11077 ) & 1533 \\
\hline Cl_03189 & - & Transposase DDE domain, Tnp1, IS1380 & pfam01609 & contig 28 (1417 to 2532$)$ & 1116 \\
\hline Cl_03190 & COG4644 & Transposase and inactivated derivatives, TnpA family, Tn3 & pfam01526 & contig 28 (4206 to 5753 ) & 1530 \\
\hline Cl_03209 & COG4644 & Transposase and inactivated derivatives, TnpA family, Tn3 & pfam01526 & contig 33 (10984 to 12801) & 1818 \\
\hline Cl_03210 & COG3316 & Transposase and inactivated derivatives, IS6 & pfam13610 & contig 33 (12848 to 13552) & 705 \\
\hline Cl_03211 & COG2801 & Transposase and inactivated derivatives, IS3_IS150 & pfam13276 & contig 33 (14010 to 13498) & 513 \\
\hline Cl_03212 & COG2963 & Transposase and inactivated derivatives, Tnp1, IS3 & pfam01527 & contig 33 (14489 to 14343 ) & 147 \\
\hline Cl_03214 & COG3316 & Transposase and inactivated derivatives, IS6 & pfam13610 & contig 34 (391 to 116) & 276 \\
\hline Cl_03235 & - & Transposase, Tnp1, IS5_IS903 & pfam13737 & contig 39 (1373 to 2212$)$ & 840 \\
\hline Cl_03261 & - & Transposase DDE domain, Tnp1, IS4 & pfam01609 & contig 51 (1 to 1188) & 1188 \\
\hline Cl_03204 & - & Mobile element protein & - & contig 33 (530 to 366) & 165 \\
\hline peg.1244 & - & Mobile element protein & - & contig 28 (149 to 742 ) & 594 \\
\hline Cl_03063 & - & Mobile element protein & - & contig 15 (32765 to 34252) & 1488 \\
\hline peg.807 & - & Resolvase-like & - & contig 15 (6452 to 6847) & 396 \\
\hline peg.1245 & - & Tn1 transposase & - & contig 28 (741 to 1088) & 348 \\
\hline Cl_03045 & - & Gifsy-2 prophage protein & - & contig 15 (7251 to 7544$)$ & 294 \\
\hline Cl_03251 & - & putative bacteriophage protein & - & contig 45 (3200 to 4171 ) & 972 \\
\hline Cl_01918 & - & $\begin{array}{l}\text { elements of external origin; phage-related } \\
\text { functions and prophages }\end{array}$ & - & contig 6 (14793 to15281) & 489 \\
\hline
\end{tabular}

\section{Genomic islands}

The analysis of the LMG 26808 genome showed that at least 12 regions with lower GC contents distributed among different contigs could be distinguished (Additional file 1: Table S3). Parts of genomic islands 3 and 4 found in LMG 26808 partly overlap with the chp region of pathogenicity island (PAI) described previously in Cmm NCPPB 382 (Additional file 1: Table S3). Genomic islands with lower GC\% are thought to be integrative elements that exhibit different codon usage relative to the rest of the genome, encode for transposases, integrases and are typically found at tRNA loci. Their acquisition is mostly a result of actions of phages, transposons or horizontal gene transfer [29]. Some of the genes present in these regions in LMG 26808 were detected previously in the genome of $\mathrm{Cmm} \mathrm{NCPPB}$ 382 but the majority represents regions that were not found in Clavibacter subspecies. The total size of these regions accounts for $265 \mathrm{~kb}(\sim 7 \%$ of the genome size). The equivalent of PAI of Cmm NCBI $382(130 \mathrm{~kb})$ containing two regions chp and tomA with important genes responsible for effective plant colonization, was not found in LMG 26808, nor in other Clavibacter genomes. However, a number of orthologs were found in all three Clavibacter genomes (Additional file 1: Table S4). The higher number of orthologs of genes encoded on chp and tomA regions (as detected by OrthoMCL) was found in Cms ATCC 33113 (32), followed by LMG 26808 (17) and Cmn NCPPB 2581 (10). Only six orthologs of PAI (chp region) found in LMG 26808 were located on the low GC region 3 and 4 (Additional file 1: Table S3).

Genomic regions with lower GC content can contain diverse genes exhibiting functions in many metabolic processes. The longest region found in LMG 26808 (more than $100 \mathrm{~kb}$ ) included genes coding for antibiotic 
resistance (beta-lactamase class A, Cl_03208, Cl_03230), transposases (Cl_03209, Cl_03212) and many hypothetical proteins (Cl_03223, Cl_03183) some of which showed the highest similarity on the protein level to genes found on Klebsiella pneumoniae plasmids. Genomic region 3 contains some genes that showed similarities to the genes found in pCM2 plasmid of Cmm NCPPB 382. The majority of them are hypothetical proteins and two of them code for acetyltransferases Additional file 1: Table S3. Previous studies indicated that some pathogenic Clavibacter strains lacking pCM1 and pCM2 showed a positive signal in hybridization experiments with specific plasmid regions of Cmm NCPPB 382 implying that some of the genes found originally in $\mathrm{Cmm}$ plasmids may be actually chromosomally-encoded in other Cmm strains [30].

Genomic regions 7, 9 and 10 with lower GC content contained some genes encoding transposases and recombinases, which might imply their possible exchange/ mobilization ability. In region 7 one phage-related gene (Cl_03043), showing homology to prophage protein gp49, was detected. Its presence may represent the remains of prophage genes.

\section{Plasmid content}

LMG 26808 did not contain any of two known virulence plasmids found in pathogenic Cmm NCPPB 382. However, the plasmid extraction demonstrated the presence of one plasmid, which size was slightly smaller than that of plasmid pCM2 (70 kb). Initially, we assumed that it might be a pCM2 that lost some genes because in the previous study we could not detect the presence of the pat-1 gene, which is encoded on the Cmm plasmid [4]. Even though we did not detect the complete pCM2, some of the genes originally encoded on this plasmid were found in LMG 26808 (Additional file 1: Table S5). Except for two genes involved in the putative conjugal transfer (pCM2_0013 and pCM_0019, coding for TraA and TraG, respectively), all the remaining genes showed to code for hypothetical or putative secreted proteins. All of them were detected on contig 6 but the order in which they were found in LMG 26808 did not match the order demonstrated in pCM2. Moreover, there are more genes present on contig 6 , some of which showed to be homologous to proteins from the $\mathrm{Cmm}$ chromosome (Cl_01961-Cmm_02708, Cl_01957-Cmm_01374). These observations may suggest that some of these plasmid genes were incorporated in the genome of LMG 26808.

The observation that some genes from pCM2 that were expressed during tomato infection by $\mathrm{Cmm}$ [31] had orthologs found in LMG 26808, might suggest that although their function is unknown, they may be essential for non-pathogenic Clavibacter as well as pathogenic $\mathrm{Cmm}$ strains (Additional file 1: Table S5). Further investigation is needed to elucidate the exact functions of these genes. The smaller plasmid pCM1 was not detected during the plasmid extraction, nor were its sequences found in the genome sequence of LMG 26808. Despite that two DNA fragments of LMG 26808 showed to be highly similar to two plasmid-encoded genes, namely pCM1_0018 and pCM1_0020, the reciprocal BLAST search revealed that these sequences from the non-pathogenic Clavibacter genome are more similar to the chromosomally encoded CMM_1065 and CMM_2443, respectively. Interestingly, the latter gene encodes CelB, which is a putative secreted cellulase that contains a cellulose-binding domain (endo1,4-beta-glucanase). Chromosomally encoded celB misses one of three protein coding domains present in the $\operatorname{cel} A$ gene. The missing endoglucanase $\mathrm{C}$-terminal domain is similar to the $\alpha$-expansin protein family that occurs in plants and is essential for development of wilting and for degradation of crystalline cellulose $[8,32]$. The lack or disruption of any of these domains of celA inevitably led to the disability to induce disease symptoms in a tomato plant [32].

Surprisingly, the genome analysis showed the presence of sequences found in Klebsiella pneumoniae plasmid pOXA-48 (61881 bp). The presence of sequences from a plasmid of Gram-negative bacteria in a Gram-positive Clavibacter strain is rather unusual and has not been reported previously. Although the genome sequence of LMG 26808 is only a draft and therefore incomplete, we could not detect any sequences that could be attributed to a Klebsiella pneumoniae Kp11978 chromosome.

The exchange of genetic material between various prokaryotes is well known and has been extensively studied over the last few decades [33-37]. Although it was demonstrated for bacteria that the gene exchange is observed more frequently between closely related genera with a similar GC content and exhibiting high sequence similarities there are examples of recent gene transfers between distantly related bacteria (e.g. Actinobacteria and gammaproteobacteria) [38].

Conjugational transfer is considered the most efficient way of LGT $[39,40]$ that contribute the most to the spread of antibiotic resistance among different bacteria [41]. This type of LGT is widely encountered among various bacterial species and even between bacteria and Archaea [42] on the one hand and between bacteria and higher organisms such as Saccharomyces cerevisiae [43], or plants [44] on the other hand. Conjugational plasmid exchange was also observed within the genus Clavibacter in which the endophytic CMM100 strain (cured of pCM1 and pCM2 plasmids) was able to acquire these plasmids from other pathogenic $\mathrm{Cmm}$ strains and restore pathogenicity [45]. Furthermore, transformation experiments carried out with Clavibacter xyli subsp. cynodontis (currently reclassified to the genus Leifsonia) 
demonstrated the possibility to acquire an IncP plasmid from Enterobacteriaceae by this Gram-positive Actinobacteria, which provided another evidence of conjugational transfer between diverse taxa [46].

Klebsiella pneumoniae strains were found in many important crops such as potato, maize, soybean, cotton and tomato $[47,48]$. Many of these strains carry plasmids that contain antibiotic resistance genes and possess the conjugation transfer systems which enable the gene mobilization and exchange among and outside Enterobacteriaceae and other bacterial genera [49]. Some genes encoded on the Klebsiella pneumoniae plasmids exhibit high similarities to regions found previously in Escherichia coli and Yersinia genomes, implying that there is an active genetic exchange among strains of these genera [50].

Although an acquisition by LMG 26808 of a relatively large plasmid originating most probably from a member of Enterobacteriaceae/Klebsiella relative by LMG 26808 (Additional file 1: Figure S2) was unexpected and unprecedented, a similar occurrence was previously reported by Baltrus and coworkers. They detected a recent acquisition of a megaplasmid by two cucumber isolates of Pseudomonas lachrymans [51]. It was suggested that this acquisition resulted from an important ecological shift across closely related Pseudomonas members and that the plasmid-encoded genes may be advantageous for the recipient bacteria.

As Klebsiella pneumoniae and Clavibacter strains thrive in the same environmental niche (associated with tomato) and because of examples of possible genetic material exchange between distantly related bacteria we can hypothesize that the acquisition of plasmid sequences encoding antibiotic resistance genes might provide a competitive advantage for the non-pathogenic Clavibacter strain LMG 26808.

\section{Non-pathogenic lifestyle}

Non-pathogenic Clavibacter strains from tomato seeds tested in the previous study [4] as well as other strains tested by Jacques and coworkers [3] did not introduce any disease symptoms when tested on tomato plants. Possible explanations for the non-pathogenic nature of these strains are i) the lack of two plasmids present in pathogenic $\mathrm{Cmm}$ and carrying virulence factors, ii) the absence of the pathogenicity island and iii) a significantly lower number of genes coding for extracellular hydrolytic enzymes including several important serine proteases, glycosyl hydrolases and the plant cell wall-hydrolyzing enzymes.

In pathogenic $\mathrm{Cmm}$, main virulence factors $c e l-A$ and pat-1, encoded on pCM1 and pCM2, respectively, are required to induce disease symptoms (wilting and canker) in tomato plants [52,32]. Moreover, genes coding for the production of extracellular enzymes, such as endoglucanase, polygalacturonase, xylanase, serine proteases and other secreted proteins have been implicated as possible virulence factors in recent reports [30,31,52,53]. The genome of LMG 26808 did not contain the most prominent virulence factors pat-1 and celA. Their absence may be directly linked with the absence of the $\mathrm{pCM} 1$ and $\mathrm{pCM} 2$ plasmids in the non-pathogenic Clavibacter. However, southern hybridization experiments with plasmid fragments containing virulence factors showed that in some plasmidfree pathogenic $\mathrm{Cmm}$ strains these virulence determinants had homologues on the chromosome [30].

A proteomic study of tomato-Cmm interaction identified several bacterial proteins with a putative role in signal perception, transduction and response to impulses. They belong to two-component system proteins, transcriptional regulators and other DNA binding proteins. They are believed to play a role in sensing the tomato plant environment and initiating pathways, possibly leading to disease development [31]. All putative genes encoding proteins that are probably involved in signal exchange between tomato and bacterium could be identified in the genome sequence of LMG 26808 (Additional file 1: Table S6).

As a non-pathogenic bacterium, LMG 26808 was hypothesized to contain less genomic information for hydrolytic enzymes that are known to be expressed during tomato infection with $\mathrm{Cmm}$ [31]. As expected, the most important group containing genes coding for secreted proteases from Pat-1 family was largely absent in LMG 26808 (Additional file 1: Table S6). Additional pat-1 homologues encoded on the $\mathrm{pCM} 2$ plasmid (plasmid homologs of pat-1, $p h p A$ and $p h p B$ ) were also absent. From seven genes encoding putative serine proteases chpA-chpG (chromosomal homologs of pat-1) [54] only sequences similar to $\operatorname{ch} p F$ and $\operatorname{ch} p G$ were detected (chpF and $\operatorname{ch} p G$ are orthologs with nucleic acid sequence similarity of $69.1 \%$ and amino acids sequence similarity of $68 \%$ ). Both these sequences, however, matched to the same region and a reciprocal best BLAST hit confirmed the presence of only $\operatorname{chpF}$. Interestingly, the lack of $\operatorname{ch} p G$ may be a possible explanation for the disability of LMG 26808 to produce a HR in nonhost plants since the chpG mutant in $\mathrm{Cmm}$ was unable to cause an HR in Mirabilis jalapa [8]. Moreover, the low colonization efficiency of LMG 26808 could be attributed to the lack of the $\operatorname{chpC}$ gene. A $\operatorname{chp} C$ mutation in the pathogenic Cmm NCPPB 382 resulted in a drastic reduction in colonization abilities in tomato plants $[8,55]$. Pseudogenes $\operatorname{chp} A, \operatorname{chpB}$ and chpD were not found in LMG 26808. The family of chp genes is important for plant-pathogen interaction in $\mathrm{Cmm}$, but probably also in $\mathrm{Cms}$ where four orthologs were found. Cmn genome had no orthologs of these genes.

The majority of members of secreted serine proteases of the Ppa family (PpaA-PpaJ) that are encoded in several different loci on the chromosome and on pCM1 could not be found in LMG 26808. Orthologs of ppaI 
and ppaF were found in LMG 26808. Cms ATCC 33113 contained orthologs of ppaB1, ppaB2, ppaF, ppaI, ppaA in the chromosome and $p p a C$ on pCS1 plasmid. On the contrary Cmn NCPPB 2581 had only one ortholog of ppaF. Since ppaA and $p p a C$ genes are important for plant colonization [8] and they were absent in LMG 26808, it can be another evidence why non-pathogenic Clavibacter strains are poorly colonizing tomato plants. Indeed, secreted serine proteases studied in pathogenic $\mathrm{Cmm}$ are thought to presumably facilitate the interaction between $\mathrm{Cmm}$ and its host plant and are believed to play a function in pathogenicity by a possible utilization of plant proteins [31]. Their lack might imply that interaction between LMG 26808 and tomato is actually very limited. TomA gene of Cmm NCPPB 382 (CMM_0090), encoding endo-1,4- beta galactosidase involved in detoxification of the alfa-tomatine, had orthologs in three other Clavibacter genomes. However, the similarity based on the amino acid sequence was rather low (coverage (\%)/ identity (\%): 47/24 in Cms, 47/23 in Cmn and 47/22 in LMG 26808).

Genes coding for subtilases $s b t A, s b t B$ and $s b t C$ are known to be secreted during the plant infection [31]. Orthologs of these three subtilases genes were found in all four Clavibacter genomes. Sbt proteins of $\mathrm{Cmm}$ are highly similar to different tomato subtilases, some of which have been associated with wound formation and pathogen responses [56] and may play a role in the disease development. Because they are present in the nonpathogenic Clavibacter strain their function probably cannot be solely associated with the disease development. Cellulases and pectinases are the most important enzymes degrading plant cell walls. In many bacteria genes encoding these enzymes were found to be virulence determinants [57]. In the genome of LMG 26808 genes for pectate lyases, pelA1 and pelA2 and cellulase celA were not found. However, another cellulase celB, the polygalacturonase $p g a A$ (whose substrate is pectin), xys $A$ (whose substrate is $\beta$-1,4-xylan) and an arylesterase (which hydrolyzes ester bonds between hemicelluloses and lignin) [58] were present in LMG 26808 (Additional file 1: Table S6). These findings support the thesis that the non-pathogenic Clavibacter strain is probably less efficient in digesting pectins and cellulose into simpler by-products than the pathogenic $\mathrm{Cmm}$ that is equipped with many various plant cell degrading enzymes.

Enzymes from a large group of glycosyl hydrolases (GH) which hydrolyze the glycosidic bond between two carbohydrates or between a carbohydrate and a noncarbohydrate molecule [59] were also expressed during plant infection of $\mathrm{Cmm}$. Therefore, many of them are assumed to be potential virulence factors that can hydrolyze substrates of plant origin [31]. Our results demonstrated that genes for the majority of these enzymes are present in
LMG 26808 suggesting that their function might not be restricted to disease development alone. The glycosyl hydrolases are not considered as bona fide virulence factors, but as reflecting the adaptation to the differing composition of nutrients in planta allowing the survival inside of the plant.

Very important functions involved in transport and metabolism are linked to the presence of $\mathrm{ABC}$ and other transporters that ensure the uptake of amino acids, metals, sugars, oligopeptides, etc. Some of these transporters that were expressed during tomato infection by $\mathrm{Cmm}$ may utilize plant molecules to support its metabolism. The genes found in the genome of LMG 26808 that code for transporters that are known to be expressed during plant infection by $\mathrm{Cmm}$ are listed in Additional file 1: Table S7. Interestingly, only five orthologs of fifty seven transporters could not be found in LMG 26808. Furthermore, the genome of non-pathogenic Clavibacter contained additional transporters that were not present in the pathogenic Cmm genome (Additional file 1: Table S2). Some of them are supposed to play a role in the active drug transport and cell protection from toxic metabolites (C_03094 and Cl_03219). Another very important example of additional ABC transporters in the genome of LMG 26808 (not found in other three Clavibacter genomes) are transporters involved in iron complex transport (ABC-type $\mathrm{Fe}^{3+}$ siderophore transport system Cl_02679 and ABC-type cobalamin/Fe3 + -siderophores Cl_ 02677) (Additional file 1: Table S8). An alternative iron uptake system found in LMG 26808 might be advantageous in an iron deficient environment. This data suggests that LMG 26808 is probably able to utilize a broad variety of compounds to maximize its survival changes. Many environmental bacteria were shown to contain a high number of transporter genes in support of an environmental lifestyle [60].

Observations described above correlate well with the initial assumptions that suggested that non-pathogenic Clavibacter strains must have lost or never contained prominent virulence determinants responsible for disease induction in tomato plants. These hypotheses were partially underpinned by similar findings in another draft genome of non-pathogenic Clavibacter LMG 26811, which lacks the majority of virulence factors including two main determinants. It also contained less plant cell degrading enzymes than pathogenic Cmm NCPPB 382 (data not shown). Furthermore, the comparative genome analysis of LMG 26808 and Cmm NCPPB 382 revealed that some putative virulence factors, determined based on expression levels obtained from the proteomic study of tomato-Cmm interaction [31], were also present in LMG 26808, which may indicate their redundant functions and suggest that they are not critical for $\mathrm{Cmm}$ virulence. 


\section{Survival in the environmental niche}

Non-pathogenic Clavibacter strain LMG 26808 was isolated from tomato seeds yet knowledge on its environmental niche is largely lacking. Ecological niche(s) and transmission routes have not yet been found. Preliminary results with colonization experiments showed poor colonization of vascular tissues of tomato and seemingly lower survival potential of LMG 26808 in comparison to Cmm [4]. The HR was not induced in Mirabilis jalapa (J. Van Vaerenbergh, data not published), indicating that non-pathogenic Clavibacter strains do not contain genes that would be recognized by the plant to trigger the active plant defense response.

Antibiotic resistance. In the genome of LMG 26808 several additional genes responsible for antibiotic resistance were detected (Additional file 1: Table S2). They coded for beta-lactamases (Cl_03263, peg.1233, peg.1766, peg.1776), chloramphenicol acetyltransferase (Cl_03044) and tetracycline efflux protein TetA (peg.1764). They showed the highest similarity to genes found in Klebsiella pneumonia, Escherichia coli and Salmonella enterica suggesting that they could originate from these bacteria. In addition to the above genes, the genome of LMG 26808 contains two drug efflux transporters (Cl_03219, Cl_03094) not found in pathogenic Cmm NCPPB 382. Interestingly, LMG 26808 contained glyoxalase/bleomycin resistance protein (Cl_03100), which probably constitutes the resistance to bleomycin-antibiotic produced by some Streptomyces strains [61].

The presence of additional acetyltransferases might suggest that LMG 26808 exhibits broad resistance to certain antibiotics as some of the acetyltransferases (GNAT superfamily) catalyze the selective acetylation of one of the four amino groups found on a diverse set of aminoglycosides with antibiotic properties [62]. Acetylation reduces the affinity of these compounds for the acceptor tRNA site on the $30 \mathrm{~S}$ ribosome. As a consequence, bacteria expressing these genes are resistant to some aminoglycosides with antibiotic properties. The ability to cope with antibiotics produced by organisms with which non-pathogenic Clavibacter strains share the environmental niche is a significant adaptive advantage. The growth of Clavibacter strains in culture is often inhibited by other faster growing organisms. Therefore, the presence of genes coding for antibiotic resistance might be the reason why nonpathogenic Clavibacter strains are more frequently encountered and isolated from the semi-specific medium during the tomato seed certification.

Toxin-antitoxin system. The presence of the toxinantitoxin (T-A) genes (YefM Cl_00198, peg.1235 and YoeB Cl_00197) in the genome of non-pathogenic Clavibacter is intriguing and raises questions concerning their origin and potential functions in relation to the physiology of the bacterium (Additional file 1: Table S2). The YefM and YoeB T-A genes were found in many bacterial genomes and sometimes more than one copy per genome [63]. It was demonstrated that $\mathrm{T}$-A systems are present only in environmental and free-living organisms and were not detected in intracellular bacteria [63]. The BLASTp analysis of YefM and YoeB genes from LMG 26808 revealed high similarities to proteins from Rhodococcus pyridinivorans AK37 and Microbacterium testaceum StLB037, respectively. The T-A system found in LMG 26808 was not present in the pathogenic Cmm, but YefM (peg.1235) was present in another non-pathogenic Clavibacter strain LMG 26811 (data not shown). Interestingly, Cmn contained another putative toxin-antitoxin system. T-A systems are not essential for normal cell growth, nevertheless they are present in many bacteria and Archaea [63]. Based on the frequency of T-A systems, it was suggested that they play subtle roles that are advantageous for cell survival in their natural habitats. Toxins may facilitate cellular adaptation of an organism to changing environments by slowing down its cell growth, inhibiting its cell growth, or causing some of its cells to die [64]. It is possible that the presence of a T-A system in the LMG 26808 genome increases the fitness of this bacterium in the occupied environmental niche. Differences in the detected toxinantitoxin systems in particular Clavibacter subspecies might be attributed to different ecological niches and inhabited hosts.

Error prone UmuDC operon. SOS mutagenesis response in bacteria includes error-prone and error-free DNA damage repair responses that are activated after exposure to different antibiotics, chemical compounds or radiation [65]. In Escherichia coli UmuDC proteins are involved in error-prone bypass of UV lesions and UmuC proteins possess DNA polymerase activity. In the SOS process many genes get induced and their products are involved in DNA repair, replication and cell cycle control in order to repair the DNA damage [66]. The genes coding for this operon were found in LMG 26808 and also in another nonpathogenic Clavibacter, LMG 26811 (data not shown), implying that their cells might have higher abilities to recover after exposures to UV and/or other types of chemicals retrieved during the seed certification procedures. Some of the sequences coding for genes of UmuC operon (e.g. peg.1211) and antibiotic resistance genes (e.g. Cl_03263) described above are associated with the plasmid and/or low GC regions (Additional file 1: Table S2).

The extracellular polysaccharide (EPS). The genomes of all four analyzed Clavibacter strains contained four gene clusters involved in exopolysaccharides production (Additional file 1: Table S9). The EPS production in LMG 26808 is expected to effectively occur since all genes involved in that process are functional (no frameshifts, no pseudogenes). LMG 26808 contains almost a 
complete set of genes involved in the EPS production described in Cmm NCPPB 382. There is, however, one notable difference between pathogenic Cmm NCPPB 382 and LMG 26808. In the EPS2 of LMG 26808 the order of the genes is disrupted because they are located at different contigs. The functionality of this gene cluster is therefore unknown. Even if functional, it will probably be dependent on different regulation factors which may eventually influence the EPS production. EPS gene clusters in the pathogenic Cms underwent quite some drastic changes with disruptions by insertion elements and most likely the EPS production in Cms ATCC 33113 is limited. EPS clusters 1, 3 and 4 are also complete in Cmn NCPPB 2581. In case of the EPS cluster 2 in Cmn NCPPB 2581 there are three additional genes (CMN_00784, CMN_00787, and CMN_00792) located in between other genes (Additional file 1: Table S9). The main EPS composition of $\mathrm{Cmm}$ and $\mathrm{Cms}$ strains was determined experimentally and it showed some differences even though the general structure of a repeating unit of four sugars seems to be the same [67]. The number of hydrolases in Cms ATCC 33113 and Cmm NCPPB 382 is the same but some of them show lower similarities indicating differing substrate specificity/a different sugar incorporated. The composition of EPS in LMG 26808 was not yet experimentally determined. Even though this non-pathogenic strain showed a very similar genetic structure of EPS clusters to those of Cmm NCPPB 382 some genes have lower similarities (e.g.: polysaccharide polymerase (Wzy2-70\% similarity; Wzy1-73\% similarity); glucosyl transferases (WcmL-75\% similarity; WcqR-70\% similarity)). These findings indicate that the composition of EPS might be different between $\mathrm{Cmm}$ and non-pathogenic strains. In many bacteria the ability to produce EPS and their presence in the cell wall surface has been shown to participate in the interaction between bacteria and the environment. EPS is believed to prevent bacterial attachment to host cells which in consequence prevents the recognition of the bacteria by the host and the induction of an HR reaction [68]. In many plant pathogens EPS production prevents bacterial immobilization by host lectins and in that way allows bacteria to spread in the xylem vessels [67]. The presence of at least three functional EPS gene clusters in LMG 26808 might facilitate the EPS production in different environmental conditions and can be an advantage for non-pathogenic Clavibacter, which probably inhabits not only tomato seeds but also other environmental niches.

\section{Conclusions}

The analysis of the genome sequence of the non-pathogenic Clavibacter LMG 26808 revealed that this strain is adapted to a non-pathogenic lifestyle. This is reflected by the lack of prominent virulence factors present in pathogenic $\mathrm{Cmm}$ and by the presence of a significantly lower number of genes encoding enzymes involved in digesting plant material and extracellular proteins that are potential virulence determinants. Also, LMG 26808 contained many transport proteins and transcriptional regulators implying its capacity to utilize various compounds and to respond rapidly to a changing environment. The genome of LMG 26808 contained also a high number of $\mathrm{ABC}$ transporters and genes involved in the cell signalling (comparable to those from some free-living bacteria).

The draft genome of the non-pathogenic Clavibacter strain and the comparative analysis with other whole Clavibacter genomes provided valuable insights into the genetic bases of pathogenicity and mechanisms involved in the adaptation to host plants and to environmental niches. Our results demonstrated that some of the putative virulence factors were also present in LMG 26808, which suggests that these genes rather contribute to the general fitness (iron uptake systems, proteases) of the bacterium by increasing competitiveness and adaptive abilities in the same environment than playing a role in virulence. Whether the non-pathogenic Clavibacter strain can turn into a pathogen will depend not only on the presence of additional fitness genes that allow for efficient host colonization and adaptation, but mainly on the presence of functional virulence genes. LMG 26808 does not contain celA and pat-1, the two most important virulence factors and lacks some other important determinants contributing to the effective plant colonization and involved in cell maceration and degradation. This specific combination of features likely represents the basis of its nature as a free-living bacterium and might exhibit the possible evolutionary process that involves horizontal gene transfer and gene loss, which shaped this bacterium into a non-pathogen. Because the diversity of non-pathogenic Clavibacter strains investigated so far is much higher than observed for $\mathrm{Cmm}$ it will be very interesting to investigate more of these strains in order to reveal the common genetic features and to determine factors responsible for their non-pathogenic nature. So far, some of the genomic adaptations, such as the presence of additional antibiotic resistance genes and a toxin-antitoxin system could be confirmed in a draft genome sequence of another non-pathogenic Clavibacter strain (data unpublished). A more in-depth comparative analysis with newly sequenced Clavibacter genomes will allow generating more knowledge about underlying biology of these bacteria and enabling the selection of group-specific regions that will serve as targets for development of reliable identification primers for novel control strategies.

The availability of genome sequences of Clavibacter strains is a critical to understanding of the processes involved in the evolution of these subspecies and in gaining 
more insight into the genetic basis of their pathogenic and non-pathogenic nature. Our findings confirmed the thesis that the non-pathogenic Clavibacter strain contains specific fitness factors but lacks crucial virulence determinants, which likely contribute to its poor colonization abilities and survival in the tomato plant. The comparison of Cmm and a non-pathogenic Clavibacter strains demonstrated that it is difficult to define real virulence factors since some of the genes previously assigned as putative virulence factors for $\mathrm{Cmm}$ are also present in the nonpathogenic strain. The role of many putative virulence factors is not clear, which partially can be attributed to the functional redundancy of these genes and to the complex and not well understood processes of their regulation. In consequence, it is dependent on the environmental niche and growth conditions (pathogen inside the host versus non-pathogenic strain in the environment) whether the presence of additional factors increasing the general strain fitness will contribute to virulence.

\section{Additional file}

Additional file 1: Table S1. Genes of plasmid pOXA-48 from a Klebsiella pneumoniae (Kp) strain Kp11978 (JN626286.1) found in the genome of LMG 26808 (Based on the BLASTn and BLASTp results). Table S2: List of 299 genes found in the LMG 26808 genome but not present in $\mathrm{Cmm}$ NCPPB 382. Table S3: Low GC regions of LMG 26808 detected by IslandViewer and their orthologs in other Clavibacter genomes. Table S4: Orthologs of genes encoded on chp and tomA regions of PAl of Cmm 382 found in other Clavibacter genomes (based on OrthoMCL). Table S5: List of genes from $\mathrm{Cmm}$ plasmids pCM1 and pCM2 found in the genome of nonpathogenic Clavibacter strain LMG 26808. Table S6: A list of Cmm genes containing known and putative virulence factors and other bacterial genes that are possibly involved in functions such as signal perception and transduction and interaction with tomato plant (as described in the publication of Savidor et al. [31]) and their orthologs in other Clavibacter genomes. Table S7: The list of transporters expressed in planta during the tomato infection by $\mathrm{Cmm}$ (Savidor et al, [31]) and their homologs and orthologs in LMG 26808. Table S8: Genes of LMG 26808 involved in iron acquisition and metabolism (based on COG groups from IMG-ER) and their orthologs in other Clavibacter genomes. Table S9: The extracellular polysaccharide (EPS) gene clusters present in Clavibacter genomes. Figure S1: Percentage of the total number of genes in each functional category as defined by COG (clusters of orthologous groups). The analysis was performed in IMG-ER. Figure S2: Plasmid extraction of LMG 26808 (arrow points at the plasmid of LMG 26808).

\section{Competing interests}

The authors declare that they have no competing interests.

\section{Authors' contributions}

MM, JW and PDV conceived the study and participated in its design. JZ participated in the study design, carried out the molecular work, performed the data analysis and drafted the manuscript. PS participated in the study design, conducted the genome assembly and calculated ANI values. SB and $\mathrm{AH}$ helped in the genomic data analysis. All authors read and approved the final manuscript.

\section{Acknowledgements}

The authors would like to thank Dr. Joachim Vandroemme (Plant Sciences Unit, ILVO, Belgium) for his useful comments and suggestions on a draft version of this manuscript. This work was performed in the Seventh Framework Programme of project KBBE-2008-1-4-01 (QBOL) nr 226482 funded by the European Commission. Het Fonds Wetenschappelijk Onderzoek-Vlaanderen (FWO) is acknowledged for the postdoctoral fellowship of Pieter Stragier and the Belgian NPPO (FAW) for partially financing ILVO-research.

\section{Author details}

'Laboratory of Microbiology, Department of Biochemistry and Microbiology, Ghent University, K.L. Ledeganckstraat 35, Gent B-9000, Belgium. ²Plant Sciences Unit - Crop Protection, Institute for Agricultural and Fisheries Research - ILVO, Burg. Van Gansberghelaan 96, Merelbeke B-9820, Belgium. ${ }^{3}$ BCCM/LMG Bacteria collection - Laboratory of Microbiology Department of Biochemistry and Microbiology, Ghent University, K.L. Ledeganckstraat 35, Gent B-9000, Belgium.

Received: 18 December 2013 Accepted: 9 May 2014

Published: 22 May 2014

\section{References}

1. Zinniel DK, Lambrecht $P$, Harris NB, Feng Z, Kuczmarski D, Higley $P$, Ishimaru CA, Arunakumari A, Barletta RG, Vidaver AK: Isolation and characterization of endophytic colonizing bacteria from agronomic crops and prairie plants. App/ Environ Microbiol 2002, 68(5):2198-2208.

2. Nazina T, Grigor'yan A, Xue Y, Sokolova D, Novikova E, Tourova T, Poltaraus A, Belyaev S, Ivanov M: Phylogenetic diversity of aerobic saprotrophic bacteria isolated from the Daqing oil field. Microbiology 2002, 71(1):91-97.

3. Jacques MA, Durand K, Orgeur G, Balidas S, Fricot C, Bonneau S, Quillévéré A, Audusseau C, Olivier V, Grimault V: Phylogenetic analysis and polyphasic characterization of Clavibacter michiganensis strains isolated from tomato seeds reveal that non-pathogenic strains are distinct from C. michiganensis subsp. michiganensis. Appl Environ Microbiol 2012, 78(23):8388-8402.

4. Zaluga J, Van Vaerenbergh J, Stragier P, Maes M, De Vos P: Genetic diversity of non-pathogenic Clavibacter strains isolated from tomato seeds. Syst Appl Microbiol 2013, 36(6):426-435.

5. Zaluga J, Heylen K, Van Hoorde K, Hoste B, Van Vaerenbergh J, Maes M, De Vos P: GyrB sequence analysis and MALDI-TOF MS as identification tools for plant pathogenic Clavibacter. Syst Appl Microbiol 2011, 34(6):400-407.

6. ISHI: Method for the Detection of Clavibacter michiganensis subsp. michiganensis on Tomato seed (Version 4). International Seed Health Initiative; 2011. http://www.worldseed.org/isf/ishi_vegetable.html.

7. Kleitman F, Barash I, Burger A, Iraki N, Falah Y, Sessa G, Weinthal D, Chalupowicz L, Gartemann K, Eichenlaub R: Characterization of a Clavibacter michiganensis subsp. michiganensis population in Israel. Eur J Plant Pathol 2008, 121(4):463-475.

8. Gartemann KH, Abt B, Bekel T, Burger A, Engemann J, Flugel M, Gaigalat L, Goesmann A, Grafen I, Kalinowski J, Kaup O, Kirchner O, Krause L, Linke B, McHardy A, Meyer F, Pohle S, Ruckert C, Schneiker S, Zellermann EM, Puhler A, Eichenlaub R, Kaiser O, Bartels D: The genome sequence of the tomato-pathogenic actinomycete Clavibacter michiganensis subsp. michiganensis NCPPB382 reveals a large island involved in pathogenicity. J Bacteriol 2008, 190(6):2138-2149.

9. Bentley SD, Corton C, Brown SE, Barron A, Clark L, Doggett J, Harris B, Ormond D, Quail MA, May G, Francis D, Knudson D, Parkhill J, Ishimaru CA: Genome of the actinomycete plant pathogen Clavibacter michiganensis subsp. sepedonicus suggests recent niche adaptation. J Bacterio/ 2008, 190(6):2150-2160.

10. Jansing $H$, Rudolph K: Physiological capabilities of Clavibacter michiganensis subsp. sepedonicus and development of a semi-selective medium. Zeitschrift Fur Pflanzenkrankheiten Und Pflanzenschutz-J Plant Diseases Pro 1998, 105(6):590-601.

11. Pitcher D, Saunders N, Owen R: Rapid extraction of bacterial genomic DNA with guanidium thiocyanate. Lett Appl Microbiol 1989, 8(4):151-156.

12. Anderson DG, McKay LL: Simple and rapid method for isolating large plasmid DNA from lactic streptococci. Appl Environ Microbiol 1983, 46(3):549-552.

13. Darling AE, Mau B, Perna NT: ProgressiveMauve: multiple genome alignment with gene gain, loss and rearrangement. PLoS One 2010, 5(6):e11147.

14. Rutherford K, Parkhill J, Crook J, Horsnell T, Rice P, Rajandream M-A, Barrell B: Artemis: sequence visualization and annotation. Bioinformatics 2000, 16(10):944-945.

15. Aziz R, Bartels D, Best A, DeJongh M, Disz T, Edwards R, Formsma K, Gerdes S, Glass E, Kubal M: The RAST Server: rapid annotations using subsystems technology. BMC Genomics 2008, 9(1):75. 
16. Delcher AL, Harmon D, Kasif S, White O, Salzberg SL: Improved microbial gene identification with GLIMMER. Nucleic Acids Res 1999, 27(23):4636-4641.

17. Mavromatis K, Ivanova NN, Chen I-MA, Szeto E, Markowitz VM, Kyrpides NC: The DOE-JGI standard operating procedure for the annotations of microbial genomes. Stand Genomic Sci 2009, 1(1):63.

18. Altschul SF, Gish W, Miller W, Myers EW, Lipman DJ: Basic local alignment search tool. J Mol Biol 1990, 215(3):403-410.

19. Tamura K, Peterson D, Peterson N, Stecher G, Nei M, Kumar S: MEGA5: molecular evolutionary genetics analysis using maximum likelihood, evolutionary distance, and maximum parsimony methods. Mol Biol Evol 2011, 28(10):2731-2739.

20. Carver T, Thomson N, Bleasby A, Berriman M, Parkhill J: DNAPlotter: circular and linear interactive genome visualization. Bioinformatics 2009, 25(1):119-120.

21. Langille MG, Brinkman FS: IslandViewer: an integrated interface for computational identification and visualization of genomic islands. Bioinformatics 2009, 25(5):664-665.

22. Hsiao WW, Ung K, Aeschliman D, Bryan J, Finlay BB, Brinkman FS: Evidence of a large novel gene pool associated with prokaryotic genomic islands. PLoS Genet 2005, 1(5):e62.

23. Waack S, Keller O, Asper R, Brodag T, Damm C, Fricke WF, Surovcik K Meinicke P, Merkl R: Score-based prediction of genomic islands in prokaryotic genomes using hidden Markov models. BMC Bioinformatics 2006, 7(1):142.

24. Varani AM, Siguier P, Gourbeyre E, Charneau V, Chandler M: ISsaga is an ensemble of web-based methods for high throughput identification and semi-automatic annotation of insertion sequences in prokaryotic genomes. Genome Biol 2011, 12(3):R30.

25. Holmes DS: TnpPred: A Web Service for the Robust Prediction of Prokaryotic Transposases. Int J Genomics 2011, 2012

26. Blom J, Albaum SP, Doppmeier D, Pühler A, Vorhölter F-J, Zakrzewski M, Goesmann A: EDGAR: a software framework for the comparative analysis of prokaryotic genomes. BMC Bioinformatics 2009, 10(1):154.

27. Li L, Stoeckert CJ, Roos DS: OrthoMCL: identification of ortholog groups for eukaryotic genomes. Genome Res 2003, 13(9):2178-2189.

28. Richter M, Rosselló-Móra R: Shifting the genomic gold standard for the prokaryotic species definition. Proc Natl Acad Sci 2009, 106(45):19126-19131.

29. Juhas M, Der Meer V, Roelof J, Gaillard M, Harding RM, Hood DW, Crook DW: Genomic islands: tools of bacterial horizontal gene transfer and evolution. FEMS Microbiol Rev 2009, 33(2):376-393.

30. Meletzus D, Bermphol A, Dreier J, Eichenlaub R: Evidence for plasmid-encoded virulence factors in the phytopathogenic bacterium Clavibacter michiganensis subsp. michiganensis NCPPB382. J Bacteriol 1993, 175(7):2131.

31. Savidor A, Teper D, Gartemann KH, Eichenlaub R, Chalupowicz L, Manulis-Sasson S, Barash I, Tews H, Mayer K, Giannone RJ, Hettich RL, Sessa G: The Clavibacter michiganensis subsp. michiganensis-Tomato Interactome Reveals the Perception of Pathogen by the Host and Suggests Mechanisms of Infection. J Proteome Res 2012, 11(2):736-750.

32. Jahr H, Dreier J, Meletzus D, Bahro R, Eichenlaub R: The endo-beta-1,4-glucanase CelA of Clavibacter michiganensis subsp. michiganensis is a pathogenicity determinant required for induction of bacterial wilt of tomato. Mol Plant Microbe Interact 2000, 13(7):703-714.

33. Gevers D, Huys $G$, Swings J: In vitro conjugal transfer of tetracycline resistance from Lactobacillus isolates to other Gram-positive bacteria. FEMS Microbiol Lett 2003, 225(1):125-130.

34. Mazodier P, Davies J: Gene transfer between distantly related bacteria. Annu Rev Genet 1991, 25(1):147-171.

35. Musovic S, Oregaard G, Kroer N, Sørensen SJ: Cultivation-independent examination of horizontal transfer and host range of an IncP-1 plasmid among Gram-positive and Gram-negative bacteria indigenous to the barley rhizosphere. App/ Environ Microbiol 2006, 72(10):6687-6692.

36. Schäfer A, Kalinowski J, Simon R, Seep-Feldhaus A, Pühler A: High-frequency conjugal plasmid transfer from Gram-negative Escherichia coli to various Gram-positive coryneform bacteria. J Bacteriol 1990, 172(3):1663-1666.

37. Trieu-Cuot P, Carlier C, Martin P, Courvalin P: Plasmid transfer by conjugation from Escherichia coli to Gram-positive bacteria. FEMS Microbiol Lett 1987, 48(1):289-294.

38. Popa O, Hazkani-Covo E, Landan G, Martin W, Dagan T: Directed networks reveal genomic barriers and DNA repair bypasses to lateral gene transfer among prokaryotes. Genome Res 2011, 21(4):599-609.

39. Dahlberg C, Bergström M, Andreasen M, Christensen BB, Molin S, Hermansson M: Interspecies bacterial conjugation by plasmids from marine environments visualized by gfp expression. Mol Biol Evol 1998, 15(4):385-390.

40. Grohmann E, Muth G, Espinosa M: Conjugative plasmid transfer in Gram-positive bacteria. Microbiol Mol Biol Rev 2003, 67(2):277-301.

41. Bennett P: Plasmid encoded antibiotic resistance: acquisition and transfer of antibiotic resistance genes in bacteria. Br J Pharmacol 2008, 153(S1):S347-S357.

42. Dodsworth JA, Li L, Wei S, Hedlund BP, Leigh JA, De Figueiredo P: Interdomain conjugal transfer of DNA from bacteria to archaea. Appl Environ Microbiol 2010, 76(16):5644-5647.

43. Heinemann JA, Sprague GF Jr: Bacterial conjugative plasmids mobilize DNA transfer between bacteria and yeast. Nature 1989, 340(6230):205-209.

44. Zambryski P, Tempe J, Schell J: Transfer and function of T-DNA genes from Agrobacterium Ti and Ri plasmids in plants. Cell 1989, 56(2):193-201.

45. Eichenlaub R, Gartemann K, Burger A: Clavibacter michiganensis, a group of Gram-positive phytopathogenic bacteria. The Netherlands: Plant-associated bacteria Springer, Dordrecht; 2006:385-422.

46. Metzler MC, Zhang YP, Chen TA: Transformation of the Gram-positive bacterium Clavibacter-xyli subsp cynodontis by electroporation with plasmids from the IncP incompatibility group. J Bacterio/ 1992, 174(13):4500-4503.

47. Quadt-Hallmann A, Hallmann J, Kloepper J: Bacterial endophytes in cotton: location and interaction with other plant-associated bacteria. Can $J$ Microbiol 1997, 43(3):254-259.

48. Rosenblueth M, Martinez-Romero E: Bacterial endophytes and their interactions with hosts. Mol Plant Microbe Interact 2006, 19(8):827-837.

49. Zhao F, Bai J, Wu J, Liu J, Zhou M, Xia S, Wang S, Yao X, Yi H, Lin M: Sequencing and genetic variation of multidrug resistance plasmids in Klebsiella pneumoniae. PLoS One 2010, 5(4):e10141.

50. Bistué AJS, Birshan D, Tomaras AP, Dandekar M, Tran T, Newmark J, Bui D, Gupta N, Hernandez K, Sarno R: Klebsiella pneumoniae multiresistance plasmid PMET1: similarity with the Yersinia pestis plasmid $\mathrm{PCRY}$ and integrative conjugative elements. PLOS One 2008, 3(3):e1800

51. Baltrus DA, Nishimura MT, Romanchuk A, Chang JH, Mukhtar MS, Cherkis K, Roach J, Grant SR, Jones CD, Dangl JL: Dynamic evolution of pathogenicity revealed by sequencing and comparative genomics of 19 Pseudomonas syringae isolates. PLoS Pathog 2011, 7(7):e1002132.

52. Dreier J, Meletzus D, Eichenlaub R: Characterization of the plasmid encoded virulence region pat-1 of phytopathogenic Clavibacter michiganensis subsp. michiganensis. Mol Plant Microbe Interact 1997, 10(2):195-206.

53. Beimen A, Bermpohl A, Meletzus D, Eichenlaub R, Barz W: Accumulation of phenolic compounds in leaves of tomato plants after infection with Clavibacter michiganense subsp. michiganense strains differing in virulence. Zeitschrift fuer Naturforschung Sec C 1992, 47(11):898-909.

54. Gartemann KH, Kirchner O, Engemann J, Grafen I, Eichenlaub R, Burger A: Clavibacter michiganensis subsp. michiganensis: first steps in the understanding of virulence of a Gram-positive phytopathogenic bacterium. J Biotechnol 2003, 106(2-3):179-191.

55. Stork I, Gartemann KH, Burger A, Eichenlaub R: A family of serine proteases of Clavibacter michiganensis subsp. michiganensis: chpC plays a role in colonization of the host plant tomato. Mol Plant Pathol 2008, 9(5):599-608.

56. Jorda L, Coego A, Conejero V, Vera P: A genomic cluster containing four differentially regulated subtilisin-like processing protease genes is in tomato plants. J Biol Chem 1999, 274(4):2360-2365.

57. Salmond GP: Secretion of extracellular virulence factors by plant pathogenic bacteria. Annu Rev Phytopathol 1994, 32(1):181-200.

58. Borneman W, Akin D, Ljungdahl L, Wilson J, Harris P: Lignocellulose degradation by rumen fungi and bacteria: ultrastructure and cell wall degrading enzymes. Nueva York: Microbial and plant opportunities to improve lignocellulose utilization by ruminants Elsevier; 1990:325-339.

59. Henrissat B, Callebaut I, Fabrega S, Lehn P, Mornon J-P, Davies G: Conserved catalytic machinery and the prediction of a common fold for several families of glycosyl hydrolases. Proc Natl Acad SCi 1995, 92(15):7090-7094

60. Harland DN, Garmory HS, Brown KA, Titball RW: An association between ATP binding cassette systems, genome sizes and lifestyles of bacteria. Res Microbiol 2005, 156(3):434-442.

61. Semon D, Movva NR, Smith T, El Alama M, Davies J: Plasmid-determined bleomycin resistance in Staphylococcus aureus. Plasmid 1987, 17(1):46-53.

62. Vetting MW, De Carvalho LP S, Yu M, Hegde SS, Magnet S, Roderick SL, Blanchard JS: Structure and functions of the GNAT superfamily of acetyltransferases. Arch Biochem Biophys 2005, 433(1):212-226. 
63. Pandey DP, Gerdes $K$ : Toxin-antitoxin loci are highly abundant in free-living but lost from host-associated prokaryotes. Nucleic Acids Res 2005, 33(3):966-976.

64. Yamaguchi Y, Park J-H, Inouye M: Toxin-antitoxin systems in bacteria and archaea. Annu Rev Genet 2011, 45:61-79.

65. Hare JM, Bradley JA, Lin C-I, Elam TJ: Diverse responses to UV light exposure in Acinetobacter include the capacity for DNA damage-induced mutagenesis in the opportunistic pathogens Acinetobacter baumannii and Acinetobacter ursingii. Microbiology 2012, 158(Pt 3):601-611.

66. Hare JM, Perkins SN, Gregg-Jolly LA: A constitutively expressed, truncated umuDC operon regulates the recA-dependent DNA damage induction of a gene in Acinetobacter baylyi strain ADP1. Appl Environ Microbiol 2006, 72(6):4036-4043.

67. Bermpohl A, Dreier J, Bahro R, Eichenlaub R: Exopolysaccharides in the pathogenic interaction of Clavibacter michiganensis subsp. michiganensis with tomato plants. Microbiol Res 1996, 151(4):391-399.

68. Van den Bulk R, Zevenhuizen L, Cordewener J, Dons J: Characterization of the extracellular polysaccharide produced by Clavibacter michiganensis subsp. michiganensis. Phytopathology 1991, 81(6):619-623.

doi:10.1186/1471-2164-15-392

Cite this article as: Załuga et al:: Comparative genome analysis of pathogenic and non-pathogenic Clavibacter strains reveals adaptations to their lifestyle. BMC Genomics 2014 15:392.

\section{Submit your next manuscript to BioMed Central and take full advantage of:}

- Convenient online submission

- Thorough peer review

- No space constraints or color figure charges

- Immediate publication on acceptance

- Inclusion in PubMed, CAS, Scopus and Google Scholar

- Research which is freely available for redistribution 